\title{
Editorial
}

\section{The 40th International Field Emission Symposium in Nagoya (Japan),}

\section{1-5 august 1993}

The 40th International Field Emission Symposium was held last summer in Nagoya. Professors F. Okuyama from Nagoya Institute of Technology and M. Yamamoto from Osaka University were the two co-chairmen of this very well organised meeting.

It is difficult, within a few lines, to give a precise idea of a meeting where more than 150 scientists met for a week and presented 70 lectures and 160 posters. The contributions dealt with various subjects related to field emission such as Field Electron Microscopy (FEM), Field Ion Microscopy (FIM), Scanning Tunnelling Microscopy (STM), Atom Probe (AP), field sources of charged particles such as Liquid Metal Ion Sources (LMIS) and applications of these techniques. I will try to illustrate some of the scientific topics involved.

25 years after my first experience with Field Ion Microscopy, I must confess that I am still fascinated by experiments where individual atoms can be observed, displaced, removed, providing information about the crystal growth processes at the atomic scale, or conversely about surface dissolution. Two very nice experiments on iridium surfaces were presented this summer. The first one (G. Ehrlich) gave a direct view of the incorporation of iridium adatoms into a lattice, not only by diffusing on smooth facets and eventually colliding with lattice steps but also by a more complex process including direct exchange with ledge atoms. The second experiment by T.T. Tsong (Penn State University) showed in detail how the (001) surface layer of iridium could dissolve at temperatures as low as $1 / 6 T_{\mathrm{F}}$.

Surface reactions are of great interest particularly for heterogeneous catalysis. The use of FEM and FIM combined with Pulse Field Desorption Mass Spectrometry (PFDMS) furnishes a detailed knowledge of mechanisms and kinetics of surface reactions. The Fritz Haber Institute in Berlin has been very active for years in this area. A very interesting study based on a model system (decomposition of methanol on Rhodium) was also presented by N. Kruse (Zurich).

In this field of surface reactions and field desorption, the Müller Outstanding Young Scientist Medal was awarded to M.C. Reckzügel, a student of W. Drachsel from the Fritz Haber Institute, for his very clever work on field desorption of $\mathrm{H}_{3}$ and field dissociation of $\mathrm{H}_{3}^{+}$.

Atom Probe techniques have been used for many years in materials science. Particularly numerous are the studies on steels or on nickel-based superalloys. New materials such as $\mathrm{TiAl}$ intermetallic compounds are now investigated by means of AP. Three contributions were devoted to the microstructure and microchemistry of these alloys. Quantitative oxygen composition measurements were presented giving new information on the role of oxygen with respect to the ductility properties of $\mathrm{TiAl}, \mathrm{Ti}_{3} \mathrm{Al}$ alloys. Another new field also opened with the capability of AP to investigate sputtered magnetic thin films as was demonstrated by Hono from IMR (Sendai).

The recent emergence of three-dimensional atom probes has been an important event in our community as well as in materials science. The first generation of instruments (PoSAP for Position Sensitive Atom Probe) was introduced by the Oxford group. The Tomographic Atom Probe (TAP a second generation instrument) developed by the French team was shown in Nagoya to be capable of giving 3-D analytic images of a metallic material in a completely quantitative way and with a spatial resolution of a few $\AA$. It is noteworthy that a session 
and a workshop were dedicated to three-dimensional atom probe recent developments. This illustrates the increasing role that such instruments have today in the Field Emission Society and the future contribution of this new tool in the investigation of processes on a near-atomic scale.

A new area, namely Vacuum Microelectronics, that is closely related to field emission has been developed extensively during the last few years. Vacuum Microelectronics continues to be dominated by Field Emitter Arrays (FEAs), the applications of which are very promising in Field Emitter Displays (FEDs) and in high frequency microwave and millimeter-wave generation.

This too short account of the $40^{\text {th }}$ IFES is of course not exhaustive and certainly somewhat subjective. I should also have talked about other contributions such as the STM observation of fullerene on copper surfaces by Hashizume and Sakurai (Sendai) or the very nice poster of Sato and Igata (University of Tokyo) with the comparison between simulation and 3-D FIM reconstruction of vacancy cascade structure, and of many other communications related to surface reactions, field emission theory.

Field emission is involved in many different domains such as fine scale, investigation of materials and microelectronics-related topics. The organizers of three related meetings have made it possible for interested scientists to attend them all next July; these are the $7^{\text {th }}$ International Vacuum Microelectronics Conference (IVMC 94) (4-7 July in Grenoble), the $13^{\text {th }}$ International Congress on Electron Microscopy 17-22 July in Paris and the $41^{\text {st }}$ Field Emission Symposium (IFES) 11-15 July organised in Rouen. These three successive weeks will certainly offer opportunities for more extensive exchanges between our scientific communities.

Alain Menand

Laboratoire de Microscopie Ionique, URA CNRS 808 Université de Rouen, Mont Saint-Aignan (France) 